\title{
Metodología de aprendizaje colaborativo para la reutilización de residuos producidos durante de la formación académica universitaria
}

Gonzalo Rodríguez-Grau ${ }^{1}$, Claudia Valderrama-Ulloa ${ }^{*}$, Catalina Sandoval ${ }^{2}$ y Felipe Vidal ${ }^{1}$

(1) Escuela de Construcción Civil, Facultad de Ingeniería, Pontificia Universidad Católica de Chile, Av. Vicuña Mackenna 4860, Macul, Santiago - Chile (correo-e: grodriguezg@uc.cl, c.valderrama@uc.cl, favidal@uc.cl).

(2) Escuela de Ingeniería, Facultad de Ingeniería, Pontificia Universidad Católica de Chile, Av. Vicuña Mackenna 4860, Macul, Santiago - Chile (correo-e: cpsandoval@uc.cl)

* Autor a quien debe ser dirigida la correspondencia

Recibido Ago. 2, 2021; Aceptado Oct. 4, 2021; Versión final Oct. 27, 2021, Publicado Feb. 2022

\section{Resumen}

Este estudio desarrolla una metodología de aprendizaje colaborativo que permite reutilizar residuos generados en la academia, sin comprometer los objetivos de aprendizaje, y fomentar en los estudiantes una actitud medioambiental responsable. La metodología tiene cinco subsecciones: 1) identificación de cursos que generan residuos físicos en los programas curriculares vigentes; 2) análisis de la factibilidad técnica de la reutilización de los desechos identificados; 3) diseño de la propuesta de intervención; 4) implementación de la metodología; y 5) evaluación de la metodología. Los resultados muestran que el aprendizaje colaborativo permite a los estudiantes de primer año aprender de la experiencia y conocimiento de estudiantes de los últimos años, impulsando una carga cognitiva mayor y más eficaz que el aprendizaje individual. Adicionalmente, se logró reutilizar el $80 \%$ de residuos generados en actividades prácticas de docencia. Se concluye que la metodología implementada concientiza a los estudiantes sobre problemáticas medioambientales (residuos) a los que se verán enfrentados cuando ya sean profesionales.

\section{Collaborative learning methodology for reusing waste produced during university education}

\begin{abstract}
This study develops a collaborative learning methodology for reusing waste generated in academia, without compromising learning objectives, while fostering environmental awareness in students. The methodology applied is divided into five sections: 1) identification of university courses that generate waste, 2) technical feasibility assessment for reusing generated waste, 3) intervention proposal design, 4) methodology's implementation, and 5) methodology's evaluation. The results show that collaborative learning allows first-year students to learn from the experience and knowledge of last-year students, promoting an increased and more adequate cognitive load than that of individual learning. In addition, $80 \%$ of the waste generated in practical teaching activities was reused. It is conclude that the methodology implemented here contributed to the creation of student awareness about environmental issues (waste) that students may face throughout their professional careers.
\end{abstract}

Keywords: university students; sustainability awareness; practical courses; product design; waste 


\section{INTRODUCCIÓN}

La crisis climática que se enfrenta hoy en día ha llevado a buscar maneras de disminuir el impacto que se genera sobre la tierra. En algunos casos se ha optado por eliminar el uso de ciertos materiales o modificar el fin que tienen, como es el caso de las bolsas plásticas de un solo uso, donde en Chile la Ley № 21.100: Chao Bolsas Plásticas (MMA, 2018) ha prohibido su entrega en el comercio. Sin embargo, existen otros desechos que se seguirán produciendo por el diario vivir y consumir de la sociedad, y resulta importante encontrar una segunda vida para ellos, fomentando una mayor eficiencia en el uso de los recursos, procurando dejar el medio ambiente en un buen estado para las generaciones futuras.

En Chile se busca que esta temática sea prioridad. En abril del 2016, el Congreso aprobó la Ley №20.920 para la gestión de residuos, la responsabilidad extendida del productor y fomento al reciclaje (MMA, 2016) poniendo los primeros límites a las empresas, donde a través de un instrumento económico de gestión de residuos, obliga a los fabricantes de ciertos productos a organizar y financiar la gestión de los residuos derivados de sus productos. En este contexto las universidades también son actores importantes en el desarrollo sustentable de la sociedad, pues enseñan y traspasan conocimientos sobre las temáticas medioambientales a las generaciones actuales. De esta manera, se vuelve primordial que las universidades tengan un comportamiento ejemplar y respetuoso con el medioambiente, transmitiendo el conocimiento necesario a los futuros profesionales para que sean capaces de promover, asegurar y luchar, en un futuro cercano, activamente sobre esta temática (Alcalá del Olmo-Fernández et al., 2021).

En esta misma línea, distintas universidades del mundo se han caracterizado por poner énfasis en el rol que cumplen las universidades en la búsqueda de soluciones a los problemas del país, poniéndose como desafío de innovar con ideas creativas y reducir su propio impacto en el medioambiente. Por ejemplo, la Universidad Politécnica de Sinaloa, México (Olaguez-Torres et al., 2019), donde se llevó a cabo un estudio preliminar el 2019 con el fin de establecer un plan de acción para minimizar los desperdicios sólidos generados dentro de la universidad. En este caso, se buscaba conocer lo que sabían los estudiantes sobre reciclaje, su gestión y tratamiento, destacando la importancia de conocer la percepción que tienen los estudiantes con respecto a las perspectivas de educación ambiental, enfocándose en fomentarla y usándola para crear estrategias de implementación dentro de las mismas actividades académicas.

La Universidad Iberoamericana de la Ciudad de México, es otro ejemplo de aquellos establecimientos que ya han comenzado a buscar reducir su impacto hacia el medioambiente. Ya desde el 2012 realizaban estudios para determinar la cantidad de residuos generados en la Universidad y poder caracterizarlos, con el fin de detectar posibles oportunidades, ya sea de reciclaje, reutilización y/o reducción (Ruiz, 2012). Por otro lado, en Venezuela, en la Universidad del Zulia, desde 2016 se busca formas para mejorar el manejo integral de residuos sólidos que tienen los núcleos universitarios (Caldera, 2016). Aquí, la opinión y percepción de los estudiantes al respecto también tiene un alto impacto, pues desde ellos nacen propuestas para mejorar el servicio y generar consciencia entre los mismos estudiantes.

En Chile, la creación de la Dirección de Sustentabilidad en el 2016 en la Pontificia Universidad Católica de Chile - UC (Pontificia Universidad Católica de Chile, 2020), entrega una señal importante de su preocupación sobre estas temáticas, integrando además la Red Campus Sustentable, una organización que reúne a distintas instituciones de educación superior chilenas que quieren actuar para la protección del medioambiente. Además, se encarga de la valorización de la sustentabilidad, realizando buenas prácticas e incorporando principios y valores en la formación académica. Este tipo de iniciativas realiza un seguimiento a la energía eléctrica, agua, transporte y residuos generados en el campus, en especial aquellos de mayor impacto, como los de lenta degradación, en particular los sólidos inertes que se acumulan en vertederos, basurales o rellenos sanitarios. De esta forma, se busca la reducción, reutilización o, como última instancia, reciclaje de todos sus residuos.

Así como la UC, hay otras universidades en el mundo que han implementado planes de manejo de residuos, que pueden resultar en una disminución de hasta un $80 \%$ de estos (Vargas et al., 2015; Dahlawi y El Sharkawy, 2021; UC, 2020), por lo que su implementación podría traer grandes beneficios a la comunidad. Estos planes y mejoras están dirigidos a los residuos generales producidos por los diferentes campus, los desechos que forman parte del funcionamiento diario de los establecimientos. Los planes pretenden disminuir la cantidad de desecho producidos y tienen el potencial de hacerse cargo de gran parte de los residuos resultantes (Torrijos, 2021; Nolasco et al., 2021; Uyanik et al., 2021). Sin embargo, existe un foco de generación de residuos que no entra en esta categoría, se trata de los residuos sólidos inertes generados en la formación de los estudiantes, es decir aquellos desechos que vienen de las actividades con fines académicos. La generación de estos residuos viene dada por la necesidad de enseñar y educar mediante actividades prácticas, donde se requiere, por ejemplo, la elaboración de hormigón o el uso de madera. Aquí se presenta una oportunidad que no se ha explorado, se busca ir un paso más allá en el compromiso con la 
sustentabilidad, encontrando una segunda vida útil para estos residuos generados, también, dentro de la universidad.

Por otra parte, el sector de la construcción en el mundo contribuye en un $40 \%$ al total de emisiones de dióxido de carbono, 36\% de energía (Huang et al., 2018), consume 50\% de los recursos naturales, $16 \%$ del consumo de agua y produce $50 \%$ de los residuos totales generados por un país (Aldana y Serpell, 2016). En Chile las cifras no son tan distintas ya que el sector construcción aporta con un 33\% al total de emisiones de dióxido de carbono (Kairies-Alvarado, 2021), consume 50\% de los recursos naturales, 26\% de energía, 6\% del consumo de agua (MINVU, 2018) y produce el $37 \%$ de los residuos totales generados en el país (Bravo et al., 2019). Es para este sector, entonces, que la Escuela de Construcción Civil UC prepara futuros profesionales que demuestren un compromiso ético y social con las personas y con su entorno, contribuyendo así con el desarrollo sustentable del sector (Escuela UC, 2014). Bajo ese compromiso, la Escuela de Construcción Civil en el 2012, suscribió el Acuerdo de Producción Limpia de la Universidad, este documento, creado por el Consejo Nacional de Producción Limpia, es un instrumento medioambiental de gestión para promover la producción limpia a través de estrategias preventivas en productos y procesos de trabajo para poder minimizar emisiones, reducir riesgos para la salud humana y ambiental, y así mejorar la competitividad. De esta manera, el año 2016 la Escuela de Construcción Civil UC fue reconocida con la certificación del Acuerdo de Producción Limpia.

Considerando este contexto y conscientes de la problemática que se describió anteriormente, la Escuela de Construcción Civil UC desea realizar, a través del aprendizaje colaborativo entre diferentes cursos, una metodología que se haga cargo de la generación de residuos físicos que los diferentes cursos producen en las actividades prácticas propias de sus materias. El aprendizaje colaborativo busca entregar espacios en que se puedan desarrollar tanto las habilidades individuales como las grupales (Laal y Laal, 2012), a partir de la interacción entre diferentes equipos de estudiantes, lo que permite la transmisión de conocimiento y experiencia entre ellos (Akhrif et al., 2020; Laal y Ghodsi, 2012). Cabe destacar que el aprendizaje colaborativo no aplica solo para estudiantes de pregrado, sino que puede ser aplicado en diferentes materias y niveles, donde suele llamarse trabajo colaborativo. En esta línea, se busca que aquellos cursos que no generan residuos puedan aportar desde sus contenidos académicos a idear soluciones para la reutilización de los materiales, o bien llevando a cabo las ideas propuestas por sus compañeros. De esta manera, es posible combinar las diferentes materias y estudiantes para un aprendizaje colaborativo, lo que permite mejorar el rendimiento del proceso y la productividad de los cursos (Bhat et al., 2020). Además, desde hace tiempo se ha demostrado que el sistema de apoyo entre pares permite a los estudiantes internalizar de mejor manera los contenidos y facilita la discusión y la interacción entre ellos, lo que ayuda a aprender de los conocimientos, habilidades y experiencias de los demás (Leinonen y Gazulla, 2014; Supena et al., 2021).

La presente investigación propone una metodología de aprendizaje colaborativo que 1) identifica los cursos y sus prácticas académicas que por sus características siempre generen residuos inertes, 2) analiza la factibilidad técnica de reutilización de los desechos identificados; 3) define propuestas de intervención mediante la identificación de cursos idóneos a intervenir y soluciones de reutilización de residuos. En ambos casos, se busca diseñar estrategias académicas que favorezcan la reutilización de los residuos físicos; 4) se implemente en distintos cursos procurando el mayor nivel de logro de los aprendizajes curriculares; y 5) se evalúe la efectividad de la aplicación de la metodología en la reducción de residuos físicos y en la integración del conocimiento disciplinar a través del aprendizaje colaborativo. Esta evaluación tiene como fin medir la efectividad de la reducción de residuos a largo plazo y un aprendizaje duradero que se lleve a la práctica profesional.

La metodología que acá se presenta podrá ser utilizada por otras carreras (como arte, diseño, arquitectura o aquellas que tengan prácticas de laboratorios con residuos inertes) que presenten este mismo problema de generación de residuos. De esta forma, la metodología promoverá la disminución de desechos a corto y a largo plazo, tanto en la Universidad a corto plazo haciéndose cargo de los desechos derivados de sus actividades académicas, y a largo plazo en los futuros profesionales mediante la adquisición de herramientas de análisis en el ámbito de la reutilización de los desechos y de una actitud informada y responsable frente a las problemáticas medioambientales actuales.

\section{METODOLOGÍA}

La metodología de trabajo se detalla en cuatro subsecciones: i) identificación de cursos que generan residuos físicos en los programas curriculares vigentes; ii) análisis de la factibilidad técnica de la reutilización de los desechos identificados; iii) diseño de la propuesta de intervención; iv) implementación de la metodología; y v) evaluación de la metodología. 


\section{Identificación de cursos que generan residuos físicos}

La metodología (Figura 1) busca ser replicable en carreras similares, por lo que en primera instancia se debe levantar información de cada curso del currículum vigente de Construcción Civil UC, mediante la revisión de los programas de cursos, sus estrategias de aprendizaje y la opinión de los profesores asignados. En esta revisión se identifican al menos tres grupos de cursos: (1) Grupo generador de residuos inertes; (2) Grupo diseñador de solución de reutilización; (3) Grupo ejecutor de solución diseñada. Con esta clasificación se procede a confeccionar estrategias de trabajo docente, donde aquellos cursos de los grupos (2) y (3) deben enfrentar el encargo solicitado por el docente, pasando a formar parte de las evaluaciones a calificar dentro del curso.

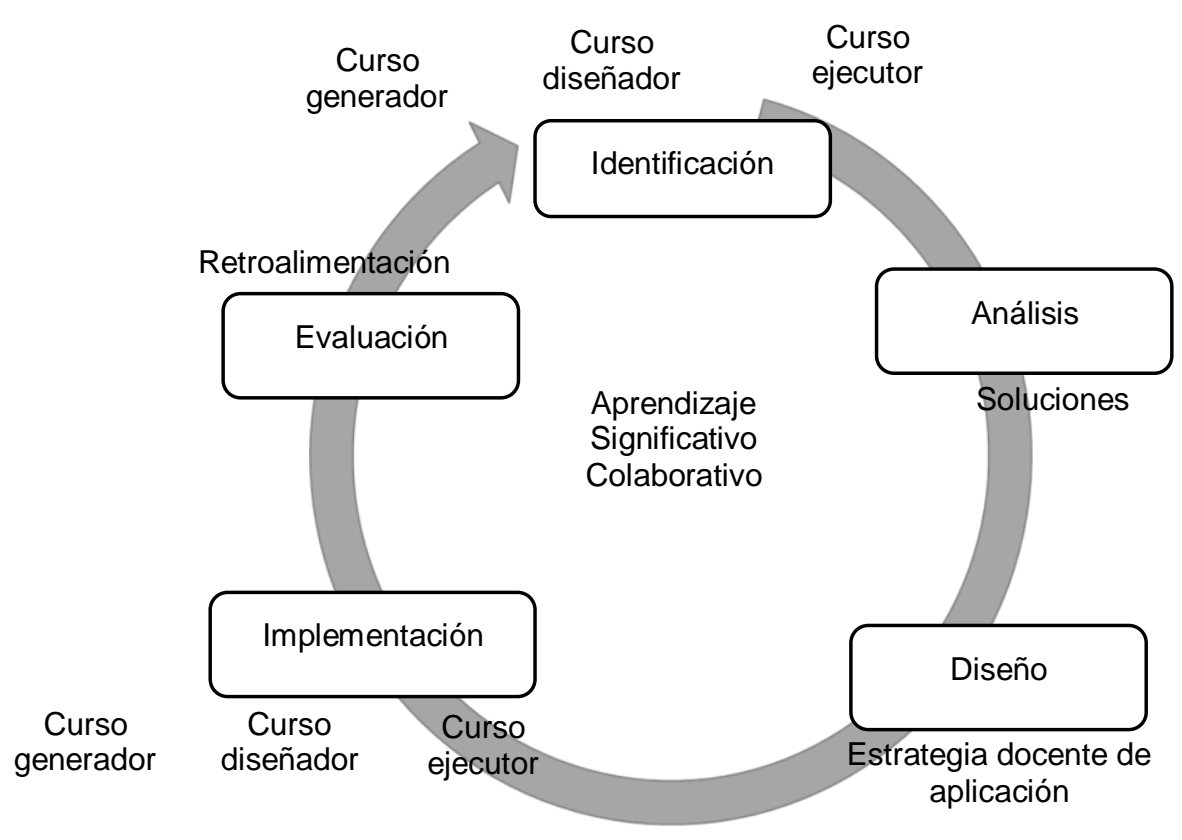

Fig. 1: Esquema metodológico

\section{Análisis de la factibilidad técnica de la reutilización}

En esta etapa se exploran las soluciones posibles para la reutilización de los residuos físicos inertes. En cada experiencia semestral se generarán una o más soluciones tangibles para la reutilización de los residuos disponibles para cada periodo. En esta primera instancia, se busca una alternativa que proponga una solución a un problema, oportunidad o necesidad que sea detectada e identificada por el mismo grupo de estudiantes del grupo diseñador de la solución. Es importante en este punto evaluar la factibilidad técnica de la reutilización de los desechos identificados, además de analizar la compatibilidad de los residuos con diversas opciones de uso. Se busca que tanto los desechos como las soluciones permitan una alternativa factible de reutilización, cubriendo una necesidad real de la Universidad y de la sociedad. Por otro lado, el diseño y la implementación de las soluciones a desarrollar serán realizadas dentro de las actividades curriculares de cada curso, además de ser parte de las tareas de aprendizaje.

\section{Diseño de la propuesta de intervención}

Se desarrollará el diseño de la propuesta de intervención a partir de los resultados de la revisión de los cursos que generen residuos, de las estrategias de enseñanzas, ya sea en talleres, laboratorios o casos de aplicación, de la revisión de sus contenidos y de los posibles cursos (generador, diseñador o ejecutor) y de las soluciones de reutilización identificadas como viables. Para ambos casos, se busca diseñar estrategias de aplicación de contenidos de los programas de los cursos, en pro de la reutilización de los residuos físicos.

\section{Implementación de la metodología}

En esta etapa la metodología se implementa, como primera instancia, en al menos un curso que genere residuos físicos y en otros cursos colaboradores (diseñador o ejecutor) en el aprendizaje, procurando el mayor nivel de logro de los aprendizajes curriculares. 


\section{Evaluación de la metodología}

Finalmente, se debe evaluar la efectividad de la aplicación de la metodología en la reducción de residuos físicos y en la integración del conocimiento disciplinar a través del aprendizaje colaborativo. La evaluación de este proceso tiene como fin lograr un aprendizaje significativo junto con la medición de efectividad en la reducción de residuos.

\section{RESULTADOS}

A continuación, se presentan los resultados obtenidos de acuerdo con la metodología presentada. En primer lugar, se identificaron los cursos generadores de residuos y aquellos que pueden apoyar con ideas para que otro curso pueda hacerse cargo de los residuos, los que se detallan en la Tabla 1.

Tabla 1: Resultados de cursos identificados

\begin{tabular}{|l|l|c|c|c|}
\hline Nombre Curso & Categoría & Nivel & $\begin{array}{c}\text { Cantidad estudiantes } \\
\text { 1er semestre }\end{array}$ & $\begin{array}{c}\text { Cantidad estudiantes } \\
\text { 2do semestre }\end{array}$ \\
\hline $\begin{array}{l}\text { Anatomía de la } \\
\text { Construcción }\end{array}$ & Colaborador diseño & 1er año & 30 & 150 \\
\hline Tecnología del Hormigón & $\begin{array}{l}\text { Generador de residuos } \\
\text { (hormigón) }\end{array}$ & 2do año & 90 & 160 \\
\hline Tecnología de Materiales & $\begin{array}{l}\text { Generador de residuos } \\
\text { (hormigón, escombro) }\end{array}$ & 1 er año & 80 & 170 \\
\hline $\begin{array}{l}\text { Formas Resistentes en } \\
\text { Madera }\end{array}$ & $\begin{array}{l}\text { Generador de residuos } \\
\text { (madera) }\end{array}$ & 5 to año & 30 & 30 \\
\hline Construcción y Sociedad & Colaborador diseño & 1 er año & 200 & 30 \\
\hline Desarrollo Sustentable & Colaborador ejecución & 5to año & 30 & 0 \\
\hline
\end{tabular}

Los cursos introductorios serán aquellos de primer año, donde se busca que los estudiantes tengan una primera aproximación a las temáticas de la carrera. En particular Anatomía de la Construcción tiene como objetivo principal distinguir diversas obras de edificación e infraestructura, su función y las partes y/o componentes que tienen. Para ello se debe interpretar planos de distintos tipos de obras de construcción y las respectivas especificaciones técnicas. Por su parte, Construcción y Sociedad busca que los estudiantes sean capaces de comprender la disciplina de la construcción y su impacto en la sociedad, reconociendo el rol y la responsabilidad del Constructor Civil en el sector. Para ello se utilizan técnicas como el dibujo a mano alzada, representación gráfica o digital, favoreciendo la interacción y el trabajo colaborativo. Ambos cursos son colaboradores de diseño, donde el aporte de cada uno depende de sus contenidos. Finalmente, la asignatura introductoria de primer año Tecnología de Materiales busca que los estudiantes puedan distinguir y analizar los principales materiales empleados en la construcción y sus propiedades. En este marco, es necesaria la generación de ciertos materiales para comprender y analizar sus propiedades, como el hormigón. Este curso entonces genera residuos, donde los estudiantes deben darle una segunda vida a los desechos de la parte práctica del aprendizaje.

Por otro lado, las otras asignaturas que se han identificado serán más focalizadas y llevarán a que estudiantes con algo más de experiencia puedan interactuar con los menos experimentados. En particular, Tecnología del Hormigón será uno de los cursos generadores de residuos, donde se tiene por objetivo comprender y analizar las propiedades del hormigón fresco y endurecido, su durabilidad y los modelos de diseño de mezcla en contextos específicos. Para llevar esto a cabo, se debe diseñar y elaborar un hormigón según la dosificación más apropiada, el cual será desechado posteriormente a las actividades de laboratorio. Sin embargo, con esta metodología, se busca que los estudiantes no lo desechen, sino que le encuentren un uso posterior y en conjunto con los cursos colaboradores se diseñe un objeto nuevo. Por su parte, Formas Resistentes en Madera también es un curso generador de residuos, en este caso de madera. Esta asignatura tiene entre sus objetivos principales analizar sistemas estructurales desarrollados con otro fin a la edificación, con madera, para ello deben experimentar los conceptos estructurales de carácter teórico mediante la fabricación de modelos y prototipos. Estos serán los que generen los desechos, sin embargo, se busca que los estudiantes puedan fabricar moldes con esta madera desechada para los modelos propuestos en hormigón.

Finalmente, el curso Desarrollo Sustentable tiene por objetivo analizar el concepto de desarrollo sustentable, su evolución en las últimas décadas y su aplicación en la edificación y el espacio público. Esta materia está dirigido a estudiantes de quinto año y se caracteriza por ser un curso colaborador de ejecución, es decir ayuda con llevar a cabo lo que ha sido diseñado y recopilado por otras asignaturas. Además, permite la interacción a estudiantes que están prontos a su egreso con estudiantes de primer año, enriqueciendo el proceso de aprendizaje. 
En segundo lugar, se evaluó la factibilidad técnica de la reutilización de los desechos identificados, para ello se definió un encargo general que analiza los antecedentes de la escuela y de los materiales desechados. De esta manera, se identifica que la cantidad de hormigón desechada por la Escuela de Construcción UC no permite la elaboración de proyectos de mayor magnitud, como podría ser la pavimentación de algún sector específico del Campus San Joaquín. Así, se busca la elaboración de inventariables, que permitan ir generando un stock semestral y que dichos objetos permitan dar solución a necesidades generadas dentro del Campus.

Los dos cursos generadores de residuos físicos inertes fueron Tecnología de Materiales y Tecnología del Hormigón. Ambos elaboran hormigón con propósitos académicos, propios al programa académico y asociados a las actividades prácticas de laboratorio. Para la confección de estos productos se utilizan betoneras móviles disponibles en el laboratorio. En el caso de Tecnología de Materiales, se realiza una actividad práctica para la aplicación del concepto de teoría de mezclas, donde se estudia la mezcla de materiales a distintas temperaturas y masas, logrando un equilibrio en su interacción con una masa homogénea a una sola temperatura. En este caso, cada grupo de estudiantes debe elaborar 40 I de hormigón, de los cuales solo se utilizan 5 I aproximadamente, para la elaboración de una probeta de $15 \times 15 \times 15 \mathrm{~cm}$ con el fin de medir la resistencia mecánica adquirida. Si se estima una cantidad promedio de 15 grupos por semestre, se obtienen aproximadamente, solo para esta actividad, 6 ton de hormigón que se está desperdiciando anualmente.

Para la elaboración de este hormigón se utiliza generalmente los siguientes ingredientes: 1) arena; 2) grava; 3) cemento; 4) agua; 5) plástico reciclado. El cemento que se utiliza es Portland comercial tipo A sin adición y agua potable para todas las mezclas. El plástico es Polietileno de Tereftalato (PET) recuperado de botellas recicladas, adicionando $0 \%, 0,5 \%$ y $1,0 \%$ en peso de los agregados a las mezclas. La relación agua-cemento corresponde a 0,52 para todas las mezclas, dosificando para una resistencia característica del hormigón de $20 \mathrm{MPa}$. En la tabla 2 se puede visualizar las proporciones de los materiales para las mezclas de hormigón.

Tabla 2: Proporciones de los materiales para las mezclas de hormigón

\begin{tabular}{|l|c|c|c|c|c|c|}
\hline \multirow{2}{*}{ Descripción } & \multicolumn{7}{|c|}{ Materiales $(\mathrm{kg})$} \\
\cline { 2 - 7 } & Agua & Cemento & Grava & Arena & $\begin{array}{c}\text { PET } \\
0,5 \%\end{array}$ & $\begin{array}{c}\text { PET } \\
1,0 \%\end{array}$ \\
\hline Por capacidad de betonera (40 litros) & 8,1 & 15,6 & 38,7 & 32,0 & 0,47 & 0,93 \\
\hline Por $\mathrm{m}^{3}$ de hormigón & 203 & 390 & 967 & 800 & 11,70 & 23,30 \\
\hline
\end{tabular}

En particular, para los cursos Construcción y Sociedad, y Anatomía de la Construcción, se realiza el encargo semestral de definir una solución para una necesidad que se pueda satisfacer con un elemento inventariable confeccionado en hormigón, al que llamaremos producto. Por su parte, para los cursos Anatomía de la Construcción y Formas Resistentes en Madera, se solicita la generación de un esquema de confección y encofrado para las posibles propuestas del producto. En los cursos de Tecnología de Materiales y Tecnología del Hormigón, y con la ayuda del curso Desarrollo Sustentable, se confeccionan y crean la o las soluciones seleccionadas del producto para el encargo o problemática definida para el semestre de trabajo. Una necesidad levantada por parte de la Dirección de Sustentabilidad de la Universidad, es la elaboración de un sistema de contención de residuos orgánicos provenientes de las áreas verdes, como hojarasca y residuos de poda, conocidos como Fracción Vegetal; y de los procesos de forestación y recuperación de suelos.

En el caso de los cursos colaboradores, los estudiantes deben investigar necesidades que se puedan solucionar con hormigón fresco en poca cantidad, y luego deben calcular una propuesta de unidad terminada y el detalle de encofrado a utilizar. En este caso el curso Anatomía de la Construcción presentó soluciones a utilizar en el Campus: bebedero para perros del campus (Figura 2a), bancas alrededor de un árbol (Figura 2b) o un diseño de mesa con asiento (Figura 2c). Otra propuesta llevada a cabo fue la realización de un ajedrez gigante, lo que permitió entregar una entretención a los mismos estudiantes para el patio, para el curso optativo de Ajedrez que dicta la Universidad y para un colegio. En este caso, se muestran en la Figura $3 a$ el encofrado optimizado utilizado y en Figura $3 \mathrm{~b}$ las piezas de ajedrez de hormigón obtenidas. Por otro lado, como un complemento al estudio se sumó un tercer curso colaborador, Desarrollo Sustentable, un curso optativo de profundización, que además es parte del programa de Magister. En dicho curso los estudiantes en el capítulo del curso "Residuos de la Construcción" debian entregar una solución de uso para el plástico. 


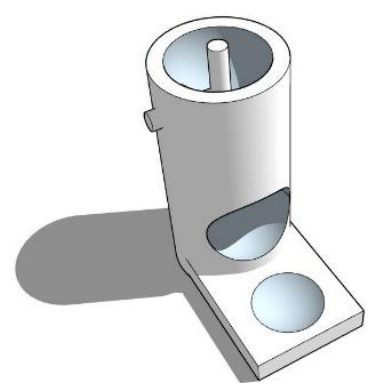

a)

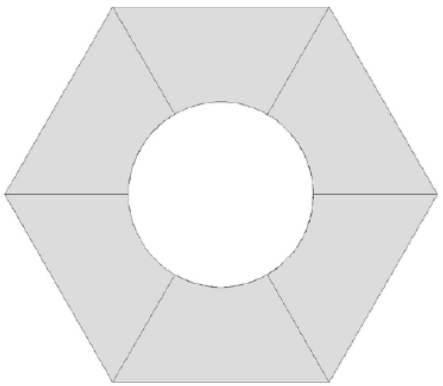

b)

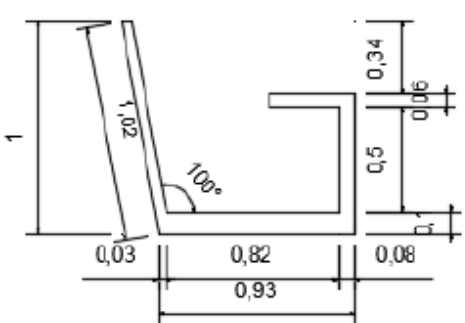

c)

Fig. 2: Algunas propuestas de productos inventariables de los estudiantes posible a crear con el hormigón fresco:

a) Bebedero de perros que viven en el campus; b) Banca alrededor de un árbol; y c) Mesa con asiento

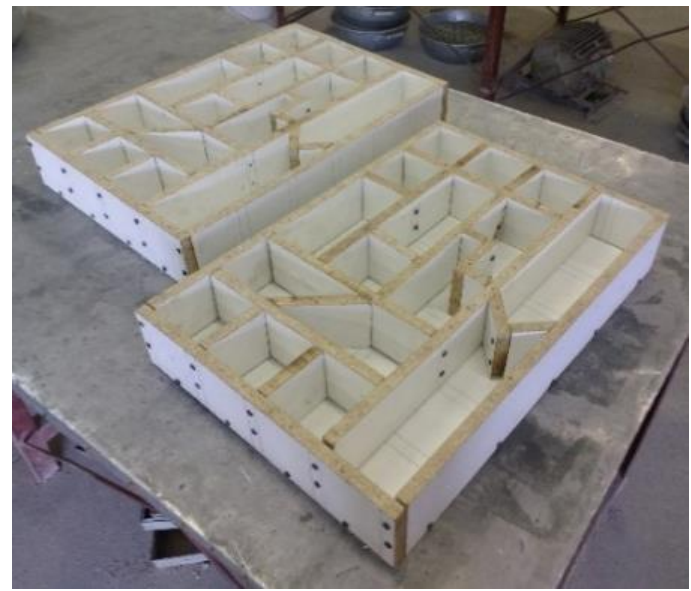

(a)

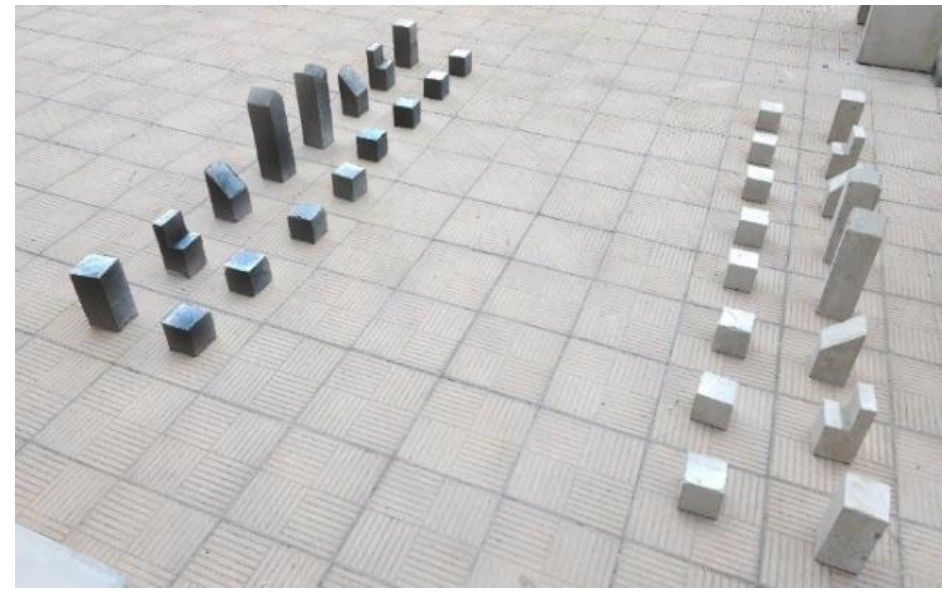

(b)

Fig. 3: (a) Encofrado optimizado para el ajedrez; (b): Piezas de ajedrez elaboradas con el hormigón reutilizado

Así, el curso ejecutor Desarrollo Sustentable, en conjunto a un estudiante de Trabajo de título, a partir el hormigón fresco y plástico reciclado de la Universidad crearon solerillas para contención de jardineras con adición de plástico y así responder al requerimiento interno de la Dirección de Sustentabilidad UC, que requería disminuir el traslado de la fracción vegetal del Campus y encontrar un lugar para disponerlo facilitando su descomposición biológica. En este entregable se ideó el diseño de la solerilla (Valderrama-Ulloa, et al., 2021), que contuviera las hojas, del encofrado con mínimos cortes y se analizaron los porcentajes aptos de Tereftalato de Polietileno (PET) para cumplir las resistencias mínimas. En la Figura 4a se observa el encofrado del diseño propuesto y en la Figura 4b las solerillas dispuestas para su instalación.

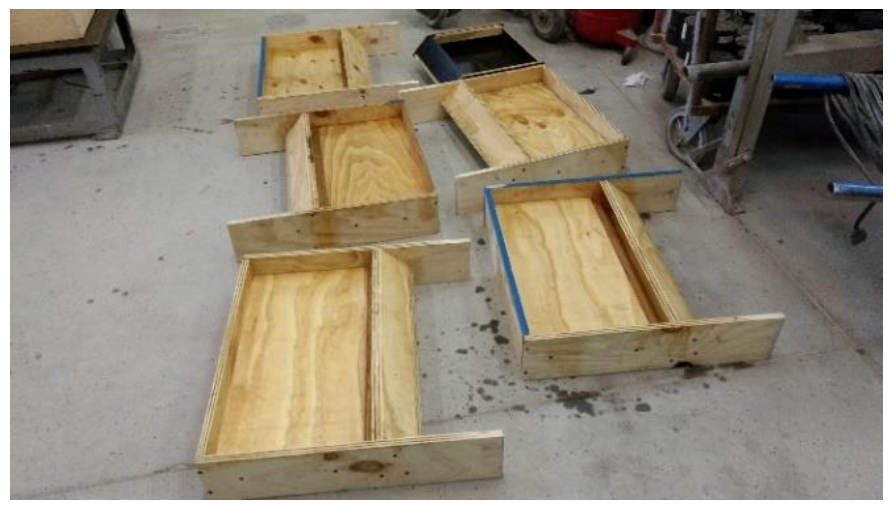

(a)

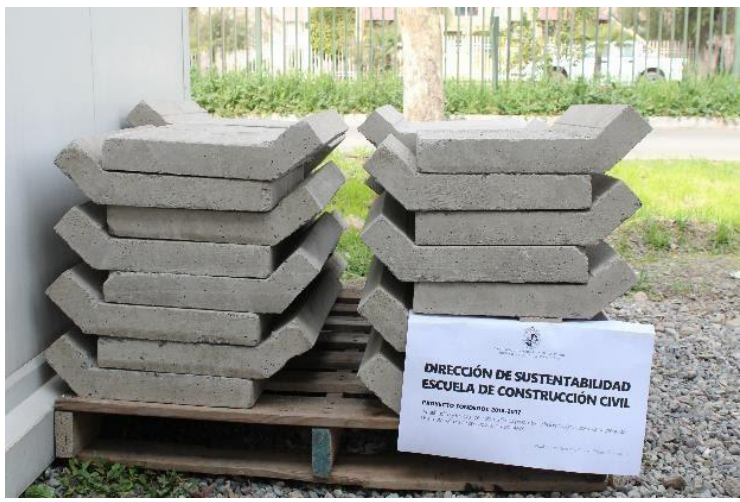

(b)

Fig. 4: (a) Encofrado optimizado; (b): Solerillas construidas en base a hormigón y PET reciclados

Finalmente, en la evaluación de las distintas actividades por parte del equipo docente y por los estudiantes, se observó la necesidad de entregar el encargo al inicio de cada curso y explicar la aplicabilidad real que tendrá el producto que diseñen o creen en el curso, y así su aprendizaje tendrá un foco para su consolidación desde un inicio, y no sólo como actividad final. Se detectó la importancia de que sea un trabajo separado en 
el tiempo, es decir avances de los productos, análisis de los productos y finalmente la ejecución del producto. La importancia de conocer desde ya las cantidades y tipos de residuos generados por docencia permite desarrollar nuevas soluciones integrables a los encargos. Desde el punto de vista del aprendizaje, los estudiantes reconocen que hablar del tema "residuos" los sensibiliza y el trabajar en casos reales aumenta su participación y responsabilidad con el curso. Además, el trabajo colaborativo permite que estudiantes de diferentes años trabajen en conjunto. Los estudiantes que vienen ingresando a la universidad sin conocimiento previo y sin experiencia profesional, colaboran para un proyecto en común con estudiantes de cursos más avanzados, con más conocimiento y experiencia, como lo son los estudiantes de tercer año e incluso quinto año quienes están ad-portas de egresar.

\section{DISCUSIÓN}

En base a los resultados obtenidos es importante recalcar la necesidad que existe de hacerse cargo de los residuos físicos generados en la formación de pregrado. Esto aplica para diversas escuelas y universidades, como la Universidad Iberoamericana de Ciudad de México, donde ya en 2012 se realizaba una cuantificación de los residuos sólidos generados, caracterizándolos (Ruiz, 2012), sin embargo, no se hace cargo de los desechos de la formación, sino de los residuos generales de su campus. En este caso, el trabajo se ha centrado en la Escuela de Construcción Civil UC. Además, resulta clave educar a los futuros profesionales del país en ámbitos como la sostenibilidad, planteando desde la formación el pensamiento crítico de estos aspectos y promoviendo la búsqueda de soluciones, ya sea para disminución o para reutilización de los desechos.

Dado que el hormigón es el principal residuo físico que se ha encontrado, es importante mencionar el potencial que presenta este para ser reutilizado, pues al encontrarse en estado fresco es posible moldearlo según las características del objeto a elaborar. De esta manera, las soluciones propuestas por los cursos colaboradores pueden diferir bastante, siendo factibles variadas formas, como las mencionadas en los resultados de la búsqueda de soluciones: solerillas y un ajedrez. En esta misma línea, es posible hacerse cargo de otros residuos, como el papel de los planos e incluso el utilizado para la realización de evaluaciones.

En particular, una de las soluciones propuestas responde a la necesidad de hacerse cargo de la fracción vegetal del Campus San Joaquín, donde esta constituye uno de los mayores volúmenes de residuos generados en la época de otoño. Otros campus universitarios fuera de Chile señalan que presentan la misma situación, donde estos residuos representan más del $20 \%$ del total de residuos generados (PUC, 2018). De aquí la importancia de buscar una solución que permita reducir estos desechos y los generados por los cursos de pregrado. En este caso, se combinan distintas problemáticas, donde la elaboración de solerillas para la recolección de la fracción vegetal es una alternativa que permite la disminución de varios residuos, el hormigón producido por los cursos de pregrado, los desechos vegetales de las áreas verdes del Campus y el plástico que se utiliza en la Universidad.

Por otro lado, se han podido implementar las ideas generadas en los cursos de diseño, donde se presentan variadas propuestas como las mostradas anteriormente. Esto permite que los cursos de ejecución puedan seleccionar las ideas que se adapten de mejor manera a las exigencias del curso y que puedan tomar lo expuesto en otras asignaturas para obtener una solución más completa. De esta forma, resulta relevante mencionar que el aprendizaje colaborativo abarca no solo diferentes asignaturas, sino que también diferentes niveles de avance curricular en la carrera. Así, los estudiantes de primer año trabajan con los de quinto año, pudiendo interactuar con estudiantes con más experiencia y conocimiento, lo que puede imponer una carga cognitiva mayor, pero es más eficaz que el aprendizaje individual, y además sus beneficios se observan a largo plazo (Zambrano et al., 2019). Por otra parte, la experiencia colaborativa mejoró el autoaprendizaje y aumentó el compromiso de los estudiantes con las actividades docentes (Cornide-Reyes y Villarroel, 2019; Rodríguez-Borges et al., 2020) y logró motivar a los estudiantes en la participación de las actividades del curso, ayudando a los profesores a mejorar los resultados evaluativos, aspectos positivos que son posibles de identificar también en el trabajo de Laal y Ghodsi (2012).

A continuación, se muestran algunos extractos de comentarios realizados por los estudiantes y profesores al término del curso. De los estudiantes: "la temática nos sensibilizó sobre los residuos", "aprendí viendo y haciendo"; "al trabajar con un estudiante más grande algún día sabré lo que él sabe"; "mi carrera impacta desde ahora" y de los profesores: "es coordinación y tiempo, pero se puede", "es una buena metodología para el aprendizaje, pero también de motivación", "nos permitió acercarnos a los estudiantes de otra forma".

\section{CONCLUSIONES}

De acuerdo con los resultados obtenidos, a la discusión y a la literatura disponible en la materia es posible concluir: 1) la metodología implementada aportó en hacer conscientes a los estudiantes sobre problemáticas 
medioambientales (residuos) a las que se verán enfrentados cuando ya sean profesionales, 2) la metodología permitió la reducción de cerca de un $80 \%$ de los residuos generados por los cursos estudiados, lo que muestra su éxito en base a sus objetivos. Esto podría aumentar si se sigue clasificando e implementando soluciones para su reducción y reutilización, 3) la metodología puede ser evolutiva, ya que se espera que los estudiantes, propongan de manera continua, ideas del cómo reutilizar los residuos sólidos y se abra oportunidades de apoyo mutuo entre el centro de estudiantes de la carrera y otras carreras para levantar necesidades locales de cada unidad y 5) también es necesario analizar restricciones curriculares de objetivos de aprendizaje en ciertos cursos, principalmente por el nivel de avance y el tiempo necesario para realizar algunas de estas actividades. Esto último puede solucionarse destinando más tiempo en la fase de identificación y análisis de las propuestas, además de controlar la programación de los cursos para que este tipo de actividad se realice al inicio del semestre.

\section{REFERENCIAS}

Akhrif, O., Benfares, C., El Bouzekri El Idrissi, Y., y Hmina, N., Collaborative Approaches in Smart Learning Environment: A Case Study, https://doi.org/10.1016/j.procs.2020.07.105, Procedia Computer Science, 175, 710-715 (2020)

Alcalá del Olmo-Fernández, M. J., Rodríguez-Jiménez, C., Santos-Villalba, M. J., y Gómez-García, G., Educar para el desarrollo sostenible en el contexto universitario: un análisis bibliométrico, http://dx.doi.org/10.4067/S071850062021000300085, Formación Universitaria, 14(3), 85-94 (2021)

Aldana, J. C., y Serpell, A., Methodology for the preparation of construction project waste management plans based on innovation and productive thinking processes: a case study in Chile, Revista de la Construcción, 15(1), 32-41 (2016)

Bhat, S., Raju, R., y otros 3 autores, Collaborative Learning for Outcome Based Engineering Education: A Lean Thinking Approach, https://doi.org/10.1016/j.procs.2020.05.134, Procedia Computer Science, 172, $927-936$ (2020)

Bravo, J., Valderrama, C., y Ossio, F., Cuantificación Económica de los Residuos de Construcción de una Edificación en Altura: Un Caso de Estudio, http://dx.doi.org/10.4067/S0718-07642019000200085, Información Tecnológica, 30(2), 8594 (2019)

Caldera, Y., Manejo integral de los residuos sólidos en un núcleo universitario, Revista Arbitrada Venezolana del Núcleo LUZ-Costa Oriental del Lago, 11, 22-36 (2016)

Cornide-Reyes, H. C., y Villarroel, R. H., Método para promover el aprendizaje colaborativo en ingeniería de software, http://dx.doi.org/10.4067/S0718-50062019000400003, Formación Universitaria, 12(4), 3-12 (2019)

Dahlawi, S., y El Sharkawy, M.F., Assessment of solid waste management practice in the university campus, https://doi.org/10.1108/IJSHE-05-2020-0183, International Journal of Sustainability in Higher Education, 22(3), 561-575 (2021)

Escuela UC, Escuela de Construcción Civil UC Programa Curricular Bachillerato, Licenciatura en Construcción y Título de Construcción Civil, https://construccioncivil.uc.cl (2014)

Huang, L., Krigsvoll, G., y otros tres autores, Carbon emission of global construction sector, https://doi.org/10.1016/j.rser.2017.06.001, Renewable and Sustainable Energy Reviews, 81, 1906-1916 (2018)

Kairies-Alvarado, D., Muñoz-Sanguinetti, C., y Martínez-Rocamora, A., Contribution of energy efficiency standards to lifecycle carbon footprint reduction in public buildings in Chile, https://doi.org/10.1016/j.enbuild.2021.110797, Energy and Buildings, 236, 1-11 (2021)

Laal, M., y Ghodsi, S. M., Benefits of collaborative learning, https://doi.org/10.1016/j.sbspro.2011.12.091, ProcediaSocial and Behavioral Sciences, 31, 486-490 (2012)

Laal, M., y Laal, M., Collaborative learning: what is it?, https://doi.org/10.1016/j.sbspro.2011.12.092, Procedia-Social and Behavioral Sciences, 31, 491-495 (2012)

Leinonen, T., y Gazulla, E. D., Design thinking and collaborative learning. Comunicar, Media Education Research Journal, 22(1), 1-14 (2014)

MMA, Ministerio del Medio Ambiente - Gobierno de Chile. Ley № 20.920: Establece marco para la gestión de residuos, la responsabilidad extendida del productor y fomento al reciclaje, http://bcn.cl/2f7b2 (2016)

MMA, Ministerio del Medio Ambiente - Gobierno de Chile. Ley № 21.100: Prohibe la entrega de bolsas plásticas de comercio en todo el territorio nacional, http://bcn.cl/2fck6 (2018)

Nolasco, E., Vieira-Duraes, P., y otros 4 autores, Characterization of solid wastes as a tool to implement waste management strategies in a university campus, https://doi.org/10.1108/IJSHE-12-2019-0358, International Journal of Sustainability in Higher Education, 22(2), 217-236 (2021)

Olaguez-Torres, E., Espino-Román, P., Acosta-Pérez, K., y Méndez-Barceló, A., Plan de Acción a Partir de la Percepción en Estudiantes de la Universidad Politécnica de Sinaloa ante el Reciclaje de Residuos Sólidos y la Educación Ambiental, https://doi.org/10.4067/s0718-50062019000300003, Formación Universitaria, 12(3), 3-14 (2019) 
Rodríguez-Borges, C. G., Bowen-Quiroz, C. A., Pérez-Rodríguez, J. A., y Rodríguez-Gámez, M., Evaluación de las capacidades de aprendizaje colaborativo adquiridas mediante el proyecto integrador de saberes,

http://dx.doi.org/10.4067/S0718-50062020000600239, Formación Universitaria, 13(6), 239-246 (2020)

Ruiz, M., Caracterización de residuos sólidos en la Universidad Iberoamericana, Ciudad de México, Revista Internacional de Contaminación Ambiental, 28(1), 93-97 (2012)

Supena, I., Darmuki, A., y Hariyadi, A., The Influence of 4C (Constructive, Critical, Creativity, Collaborative) Learning Model on Students' Learning Outcomes, https://doi.org/10.29333/iji.2021.14351a, International Journal of Instruction, 14(3), 873-892 (2021)

Torrijos, V., Dopico, D. C., y Soto, M., Integration of food waste composting and vegetable gardens in a university campus, https://doi.org/10.1016/j.jclepro.2021.128175, Journal of Cleaner Production, 315, 1-16 (2021)

UC, Pontificia Universidad Católica de Chile, Reporte de Sustentabilidad 2018-2019, Santiago, Chile (2020)

Uyanik, I., Özkan, O., y Mihçiokur, H., Waste management in a university campus, Sustainable Engineering and Innovation, 3(1), 49-53 (2021)

Valderrama-Ulloa, C., Parra, F., Marín-Uribe, C. R., y Rodríguez-Grau, G., Solerillas de hormigón y plástico reciclado para la conformación de jardineras: hacia un producto más sustentable, http://dx.doi.org/10.4067/S071807642021000200119, Información Tecnológica, 32(2), 119-130 (2021)

Vargas, O., Alvarado, E., López, C., y Cisneros, V., Plan de manejo de residuos sólidos generados en la Universidad Tecnológica de Salamanca, Revista Iberoamericana de Ciencias, 2(5), 1-9 (2015)

Zambrano, R. J., Kirschner, F., Sweller, J., y Kirschner, P. A., Effects of prior knowledge on collaborative and individual learning, https://doi.org/10.1016/j.learninstruc.2019.05.011, Learning and Instruction, 63, 1-8 (2019) 